\title{
EVIDENCIAS A CIELO ABIERTO PARA DISCUTIR SUPERFICIES POTENCIALES DE ACTIVIDAD TEMPRANA EN PATAGONIA CENTRO OCCIDENTAL (44-45 S)
}

\author{
CÉSAR MÉNDEZa \& AMALIA NUEVO-DELAUNAYa
}

\begin{abstract}
RESUMEN
Se presentan los resultados de la búsqueda guiada de evidencias tempranas a cielo abierto enmarcadas en un programa de evaluación de superficies potenciales de ocupación humana de la transición Pleistoceno-Holoceno y del Holoceno temprano. Se estudiaron áreas seleccionadas de la estepa al este de los Andes de Patagonia centro oeste. Los nuevos hallazgos corresponden a concentraciones superficiales de material lítico en los valles de Cisnes y Nirehuao. Los datos se discuten en el marco regional con el propósito de exponer nuevos aspectos a considerar dentro de las alternativas que ofrece el material de superficie y los contextos a cielo abierto.
\end{abstract}

PALABRAS CLAVE: sitios a cielo abierto, tecnología lítica, transición Pleistoceno-Holoceno, Holoceno temprano, Patagonia Centro Occidental.

\section{THE OPEN-AIR EVIDENCES FOR DISCUSSING POTENTIAL EARLY HUMAN ACTIVITY SURFACES IN CENTRAL-WESTERN PATAGONIA (44-45 S)}

\begin{abstract}
This paper presents the results of the guided search of early open-air evidence framed within a program for assessing potential surfaces for human occupations during the Pleistocene-Holocene transition and the early Holocene. We studied selected areas of the steppe located east of the Andes of central-western Patagonia. The new findings are surface concentrations of lithic material in the Cisnes and Nirehuao river valleys. The data is discussed in a regional frame with the purpose of showing new aspects to consider within the alternatives offered by surface material and open-air contexts.

KEY WORDS: open-air sites, lithic technology, Pleistocene-Holocene transition, early Holocene, Central-western Patagonia.
\end{abstract}

a Centro de Investigación en Ecosistemas de la Patagonia, José de Moraleda 16, Coyhaique. $\$ cesar.mendez@ciep.cl; amalia.nuevo@ciep.cl 


\section{INTRODUCCIÓN}

Patagonia es reconocida por algunas de las primeras evidencias concluyentes para el poblamiento inicial de Sudamérica a través del descubrimiento de las asociaciones entre artefactos de factura humana y restos de fauna del Pleistoceno en Cueva de Fell durante la década de 1930 (Bird, 1988). A partir de ahí, los restos humanos fechados sobre 10.000 años calibrados antes del presente (cal AP) al sur de los $42^{\circ} \mathrm{S}$ fueron siempre detectados en depósitos estratificados registrados en cuevas y aleros rocosos (e.g., Borrero \& Franco, 1997; Miotti \& Salemme, 2003; Borrero et al. 2019). Los depósitos bajo reparo generalmente proveen las condiciones necesarias para la supervivencia del material fechable como carbón, huesos con colágeno y otros restos orgánicos. Por lo tanto, el conocimiento arqueológico del poblamiento temprano de Patagonia está sesgado en favor de los restos recuperados dentro de cuevas; las que, a su vez, sabemos que proporcionan un conjunto limitado de evidencias. Estos sesgos han sido reconocidos desde una perspectiva geoarqueológica (Borrero, 2001; Borrero et al. 2007), así como sobre la base de aspectos del comportamiento humano esperable en este tipo de sitios (Borrero, 1989; Goñi, 1995; Jackson, 2007).

Los sitios a cielo abierto con edades del Pleistoceno final al norte de $42^{\circ} \mathrm{S}$ son relativamente comunes y demuestran que las actividades a cielo abierto de los cazadores-recolectores de dicha temporalidad pueden sobrevivir y contribuir a la variabilidad del registro arqueológico (e.g., Jackson et al. 2007; Dillehay et al. 2008; Politis et al. 2016; Suárez et al. 2018). Esto es consistente con la evidencia global de cazadores-recolectores del Pleistoceno (e.g., Gamble, 1999) y con el hecho que los campamentos al aire libre sean dominantes entre las evidencias para los primeros pobladores de América del Norte (e.g., Collins, 1991; Waters \& Stafford, 2013). Dado que la evidencia arqueológica de la transición PleistocenoHoloceno en sitios al aire libre de Patagonia aún no ha sido fechada, la interpretación de actividades al aire libre como la adquisición de materias primas líticas, la caza, el procesamiento inicial de presas, entre otras, generalmente se basa en conjeturas extrapoladas desde los conjuntos materiales excavados en cuevas (e.g., Jackson, 2002).
Las cuevas con ocupaciones que datan de la transición Pleistoceno-Holoceno en Patagonia son a menudo pequeñas (promedio: $87,5 \mathrm{~m}^{2} ; \mathrm{N}$ = 9), quizás con la notable excepción de Cueva del Medio (3567 $\mathrm{m}^{2}$; Martin et al. 2015) y como tales, no brindan las condiciones necesarias para una habitabilidad prolongada. Más bien son adecuadas para ocupaciones transitorias, lo que se ve respaldado por el tamaño y la baja inversión en la confección de los fogones (Frank, 2012; Méndez et al. 2018; Borrero et al. 2019) y las características de conjuntos óseos y líticos (e.g., Borrero \& Franco, 1997; Civalero \& Franco, 2003). El sitio Amigo Oeste en la localidad Los Dos Amigos de la Meseta de Somuncurá, donde se obtuvieron 132 puntas de proyectil de tipo "cola de pescado" de superficie, ilustra el uso de lugares al aire libre pese a que no se cuente con edades radiocarbónicas (Hermo et al. 2015). Éste se suma a numerosos puntos aislados a través de Patagonia donde cabezales de este tipo han sido registrados de forma dispersa, indicando posibles paisajes ocupados entre 12.800 y 12.100 años cal AP (Weitzel et al. 2018). Se puede afirmar una idea similar respecto a la distribución de los "litos discoidales" cuya cronología oscila en el rango entre 12.900 y 8.300 años cal AP (Jackson \& Méndez, 2007; Hermo et al. 2013). Estos artefactos pueden considerarse como un buen punto de partida para desarrollar búsquedas guiadas hacia formas del paisaje específicas (Méndez et al. 2013).

En Patagonia, al igual que en el resto de Sudamérica, algunos programas de investigación han introducido el uso de la geomorfología y la reconstrucción del paleoambiente en el desarrollo de estrategias de búsqueda guiadas (e.g., Jackson et al. 2007; McCulloch \& Morello, 2009; Santoro et al. 2011). Reconociendo factores geográficos, ambientales y procesos de formación del registro arqueológico en distintas escalas espaciales, es factible precisar las condiciones que preservarían y/o expondrían material arqueológico "temprano". A través de la combinación entre la reconstrucción de paisajes glaciares y la ubicación de los sitios previamente conocidos de la transición PleistocenoHoloceno en Patagonia Centro Occidental (PCO), definimos las condiciones más favorables para registrar dichas evidencias en los valles al oriente de la cordillera de los Andes. Se puso a prueba 
un programa de búsqueda dirigida que dio lugar al hallazgo de una punta de proyectil tipo "cola de pescado" separada por $300 \mathrm{~m}$ de un "lito discoidal", lo que remarcó el potencial del uso de la geomorfología para definir espacios al aire libre en marcos temporales específicos (Méndez et al. 2013, 2019).

En este trabajo se presenta una serie de hallazgos nuevos llevados a cabo en el marco de un proyecto de investigación que buscó evaluar la existencia de superficies a cielo abierto utilizadas durante la transición Pleistoceno-Holoceno y el Holoceno temprano en el este de PCO. Sustentados sobre los avances anteriores, se realizaron trabajos de campo (enero y febrero de 2018) en áreas y sitios que previamente mostraron potencial y que derivaron en los hallazgos de nuevas concentraciones de material lítico en superficie (Appeleg 1 y Vega Coichel). El material lítico fue analizado desde una perspectiva tecno-estilistica y comparado con evidencias regionales que permiten establecer paralelos relevantes. A fin de caracterizar las piezas líticas se incluyeron esquemas diacríticos. Este método tiene eventuales fines comparativos para establecer paralelos con otras piezas análogas. Sobre una proyección ortogonal del contorno de la pieza se graficaron las aristas en orden decreciente, iniciando con las extracciones principales y más grandes y luego, mediante flechas numeradas, se muestra la dirección de la extracción y su orden en la secuencia de talla (Inizan et al. 1999).

\section{MARCO REGIONAL AMBIENTAL Y ARQUEOLÓGICO}

PCO (entendida aquí como la región de Aisén, Chile, $43^{\circ} 40^{\prime}-49^{\circ} 15^{\prime}$ S) es una región de contrastes; donde la abrupta cordillera andina desciende directamente en el océano Pacífico y llanuras sedimentarias glacio-genéticas se extienden hacia el este. El área de estudio corresponde a las secciones superiores de dos valles ubicados al este de la cordillera de los Andes: Cisnes y Nirehuao (Fig. 1). Se caracterizan por extensos cinturones de morrenas y unidades sedimentarias glaciolacustres que han sido modeladas por la erosión fluvial Holocena. La distribución de la vegetación está controlada principalmente por una marcada gradiente de precipitación producida por la interacción entre el cinturón de vientos del oeste y la posición de la cordillera de los Andes, que produce una sombra de lluvia hacia el oriente (Garreaud, 2009). Bosques caducifolios templados de Nothofagus pumilio y Berberis ilicifolia se distribuyen en las estribaciones de los Andes orientales, transformándose gradualmente en una estepa mediterránea de Festuca pallescens y Mulinum spinosum hacia el este (Luebert \& Pliscoff, 2006).

El retiro del hielo que ocupaba las cuencas de Cisnes y Nirehuao durante el último máximo glacial (UMG) se caracterizó por la estabilización de varios frentes y la formación de lagos proglaciares de escala regional que se drenaron a través de pulsos, en un proceso que concluyó hace ca. 12.900 años atrás (García et al. 2019). El incremento en humedad efectiva al oriente de la cordillera se inició hace 14.600 años llegando a su máximo hacia los 8.000 años cal AP como lo indica la información combinada del polen fósil de los testigos sedimentarios de El Shaman y El Embudo en el valle Cisnes (De Porras et al. 2012, 2014). Los registros muestran una transición desde un ambiente de baja precipitación con pastizales abiertos, hacia una mayor expansión forestal asociada a mayor pluviosidad como consecuencia del desplazamiento de la influencia de los vientos del oeste (De Porras et al. 2012, 2014).

Si bien las fechas para el poblamiento inicial de la Patagonia oriental son ca. 13.000 años cal AP (Prates et al. 2013), PCO fue poblada casi un milenio después (Méndez, 2013; Borrero et al. 2019). La presencia humana inicial fue registrada en la estepa al oriente de la cordillera de los Andes en Cueva de la Vieja y El Chueco 1, sitios con edades máximas de 12.000 años cal AP y 11.500 años cal AP, respectivamente (Méndez et al. 2016, 2018). Muestran evidencias de ocupaciones basales de baja intensidad, con un incremento en densidad y frecuencia de las evidencias durante el Holoceno temprano, lo que está respaldado por otros sitios cercanos tales como Baño Nuevo 1 y Casa de Piedra de Roselló (Mena \& Stafford, 2006; Castro Esnal et al. 2017). El conocimiento del proceso de poblamiento inicial de PCO se funda en datos arqueológicos y bioantropológicos controlados cronológicamente en estas cuevas (Borrero et al. 2019). Sin embargo, varios trabajos han sugerido 


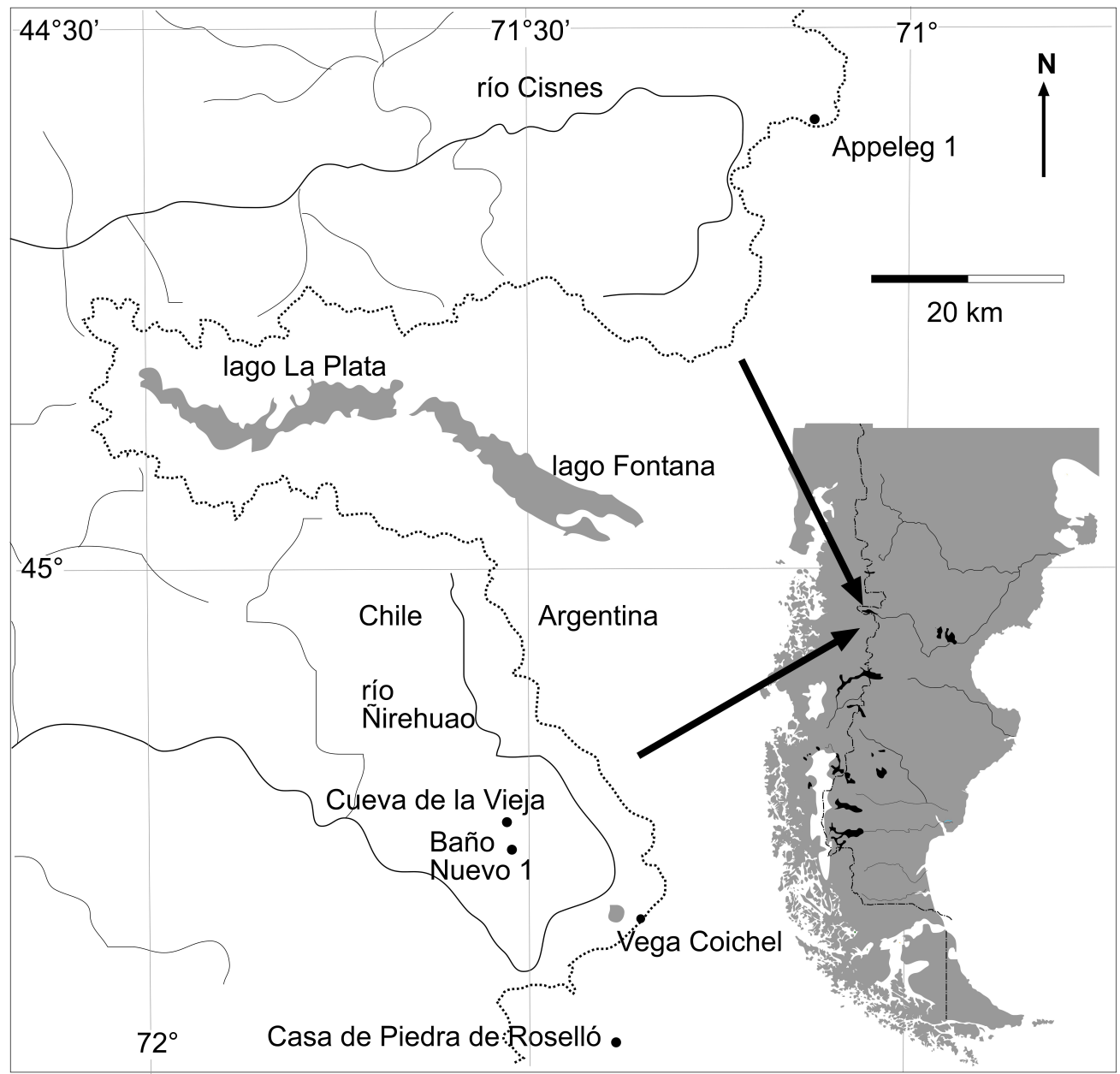

Fig. 1. Mapa del área de estudio con los sitios discutidos en este trabajo.

la posibilidad de ocupaciones a cielo abierto de edad contemporánea, aunque ninguno ha sido capaz de proveer evidencias radiocarbónicas concluyentes (e.g., Bate, 1974; Sade, 2007; Jackson \& Méndez, 2007; Méndez et al. 2013). Sólo un trabajo abordó este problema bajo una perspectiva geoarqueológica y buscó fechar unidades sedimentarias como marcadores temporales que enmarcaran la evidencia a escala espacial del sitio arqueológico (Méndez et al. 2019).

\section{LAS EVIDENCIAS ARQUEOLÓGICAS A CIELO ABIERTO}

\section{Appeleg 1}

El sitio Appeleg 1 corresponde a una serie de hoyadas de deflación sobre dunas de arenas con un área de $14.500 \mathrm{~m}^{2}$ alrededor de un parche de bosque de Nothofagus en las cabeceras del río Cisnes (Reyes et al. 2006). En su superficie se exponen concentraciones de material lítico, óseo, cerámica, vidrio y metal y, en sectores puntuales, fue posible identificar agrupaciones intencionales de piedras y áreas de combustión (Velásquez et al. 2007). Sondeos cercanos a algunas de estas áreas permitieron obtener dos edades radiocarbónicas que combinadas producen un rango entre 330-500 años cal AP (Méndez et al. 2016). Éstas se suman a otras dos edades más tempranas de termoluminiscencia de $1.290 \pm 130$ y $740 \pm 80$ años atrás (Velásquez et al. 2007). Una nueva fecha de 730-800 cal AP (917 \pm 23 BP, D-AMS 027545, carbón vegetal, pMC 89,21 $\pm 0,26)$ de un fogón excavado en la 
unidad B1 se ajusta a los rangos antes publicados. La variabilidad y atributos de la gran mayoría de las evidencias en superficie están en acuerdo con esta cronología del Holoceno tardío final (Méndez et al. 2006; Contreras et al. 2016). Sin embargo, hallazgos específicos habían llamado la atención, ya que no siguen las expectativas morfológicas del material típicamente tardío (Méndez et al. 2006; Jackson \& Méndez, 2007).

En el marco de los trabajos de campo (campaña 2018) se reconoció una nueva concentración de material lítico en superficie (App 35-36; 4435'01'S; 7107'28'O; 902 $\mathrm{msnm}$ ) cuyas características formales lo distinguen de las evidencias anteriormente recuperadas en el sitio (Méndez et al. 2006; Velásquez et al. 2007; Contreras et al. 2016). En un área inferior a $12 \mathrm{~m}^{2}$, se registró un pequeño conjunto de piezas de las cuales destacaban artefactos más grandes a la media del sitio cuyo adelgazamiento bifacial superaba su eje medio, lo que produjo secciones delgadas. En un caso se trata de una pieza fracturada, cuya porción proximal indica una morfología triangular de bordes paralelos y base ligeramente cóncava (Fig. 2b). Está manufacturada sobre un basalto de grano fino. Otras dos piezas, de diferentes rocas silíceas, también se encuentran en estado fracturado (Figs. 2d y 2e). Las materias primas son de fuente desconocida. En todos los casos se alternó ambas caras para las fases de adelgazamiento y formatizado marginal. El retoque (lascados $>2$ $\mathrm{mm}$ de ancho en la boca de lascado) se observó discontinuo y ubicado principalmente en una de las caras, aunque penetrante y en ángulo agudo, descartándose el pisoteo. El hecho que las piezas se encuentren fracturadas sugiere que la ubicación del retoque en una sola de las caras es porque su manufactura no estaba completa aún. Dentro de la concentración se recuperó también una lasca retocada de andesita, de espesor medio y con remanentes de corteza (Fig. 2a). Presenta retoque por ambos márgenes de una misma cara; éstos, sin embargo, no modificaron mayormente la forma base. Su borde activo recto y abierto y el ángulo producido por el retoque permiten clasificarla como una raedera convergente. Finalmente, a escasos metros de esta concentración se recuperó una pieza bifacial espesa de andesita en estado inicial de manufactura (Fig. 2c). Se registró sólo su porción medial (dos fracturas transversales); sin embargo, la secuencia de sus extracciones también sugiere alternancia en las caras durante el proceso de adelgazamiento bifacial.

En el caso de las tres primeras piezas bifaciales, éstas se diferencian de los conjuntos dominantes en el sitio (i.e., material típicamente tardío) por su relación tamaño/espesor (pese a encontrarse en estado fracturado son apreciablemente más grandes y delgadas) y, en el caso de la base triangular, por sus atributos tecnológicos (ausencia de pedúnculo) y la selección de la materia prima (no corresponde a una variedad silícea). Es decir, el conjunto difiere de los atributos observados en los pequeños cabezales bifaciales triangulares pedunculados característicos del sitio (Contreras et al. 2016). En el caso del artefacto de filo abierto, se diferencia de los demás conjuntos por la materia prima seleccionada, su mayor tamaño y espesor y por el hecho que el retoque no haya modificado mayormente la forma base.

\section{Vega Coichel}

Al oriente de la laguna Coichel, en la cuenca del río Nirehuao, adyacente a la frontera entre Chile y Argentina, se extiende un sistema de dunas deflacionado de $4 \mathrm{~km}^{2}$ compuesto por arenas, limos y arcillas finamente seleccionadas (Suárez et al. 2007). En una pequeña cuenca al este del sector $\left(48.900 \mathrm{~m}^{2}\right)$ se expone material arqueológico superficial en baja frecuencia. Una serie de secciones estratigráficas expuestas que resistieron la erosión, muestran unidades de limo con capas finas de cenizas volcánicas depositadas en un entorno subacuático poco profundo que intercalan con limos arcillosos muy orgánicos. Éstos sugieren la existencia de un antiguo humedal, por lo que lo hemos llamado Vega Coichel (Méndez et al. 2019). Las edades radiocarbónicas de la unidad orgánica (SU\#2) se extienden en un rango confiable entre los 7.440-7.620 y 4.230-4.420 años cal AP.

Trabajos anteriores en la localidad permitieron registrar un "lito discoidal" en superficie, a $10 \mathrm{~m}$ de uno de los perfiles expuestos, apoyando sobre el techo de la unidad estratigráfica enterrada (SU\#3) por la capa de limo orgánico 

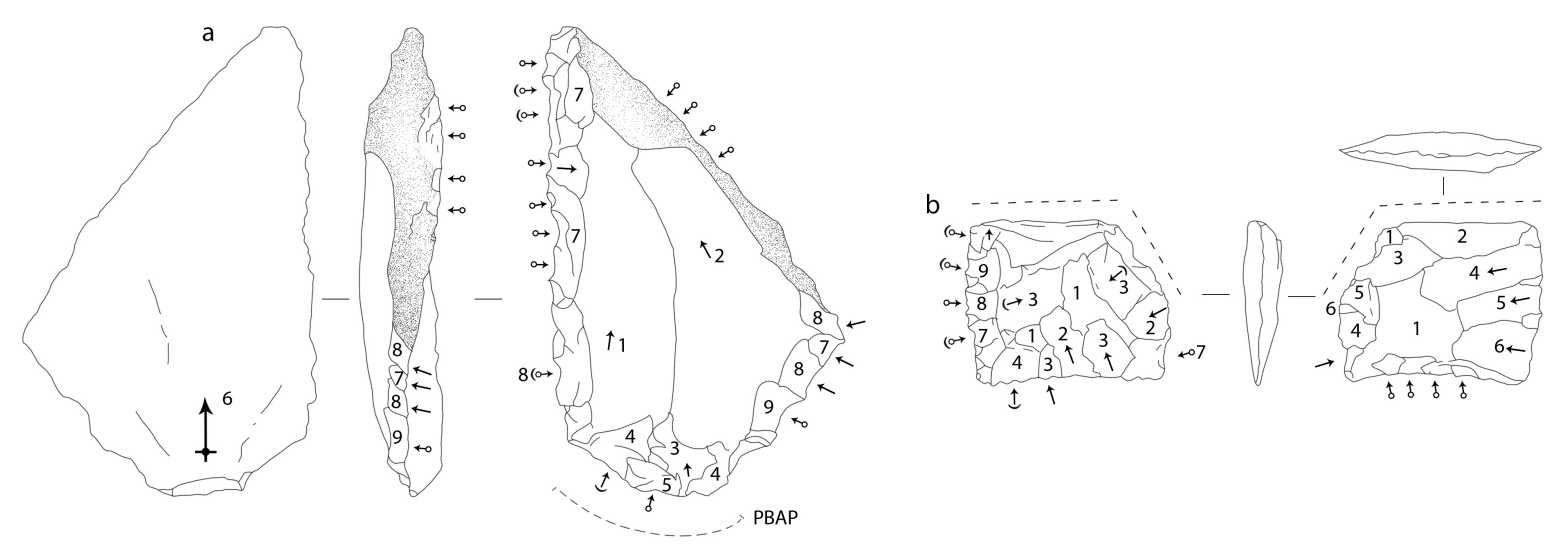

C
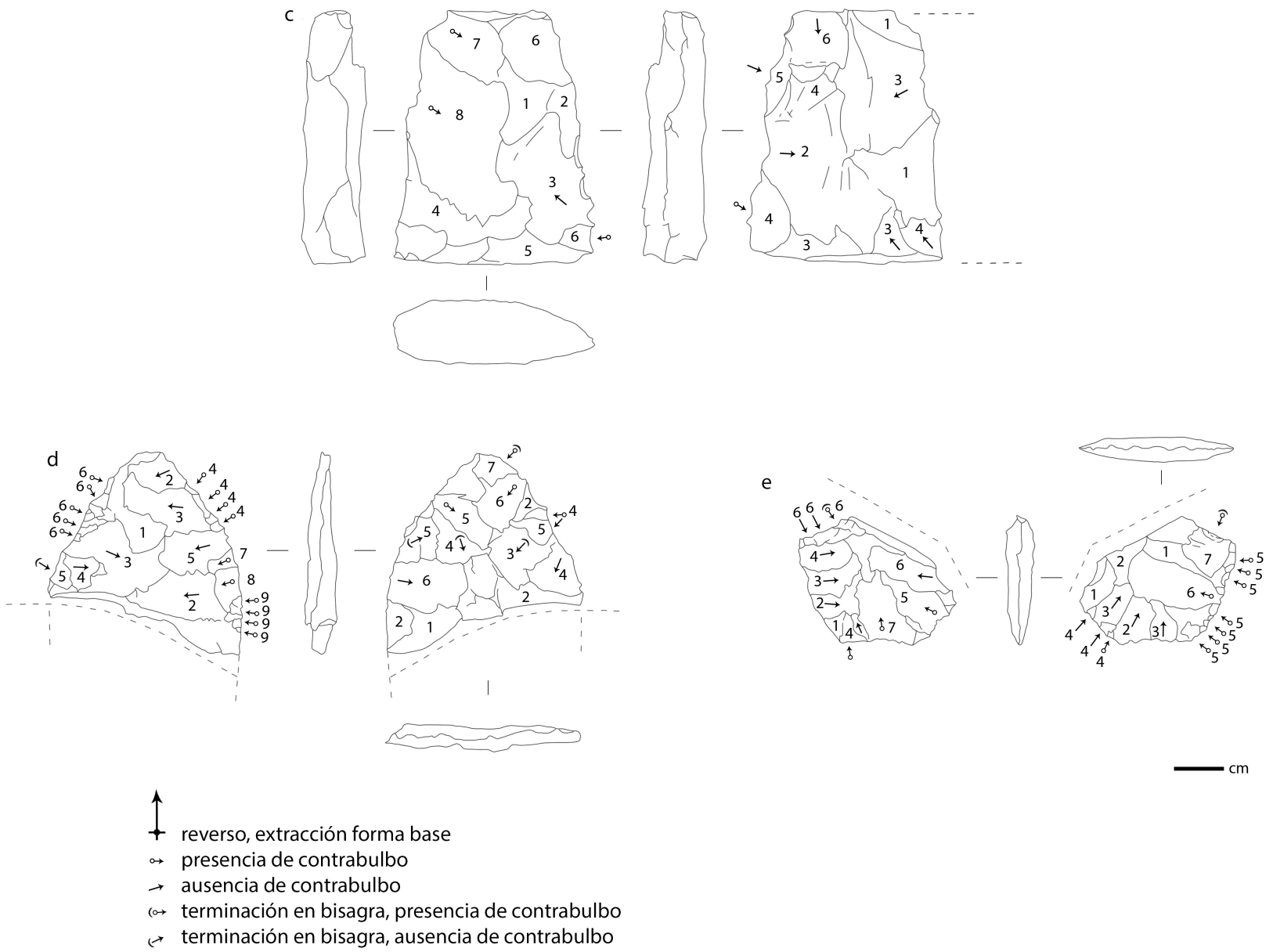

Fig. 2. Material lítico de Appeleg 1 (concentraciones App 35-36: a-b y d-e; App 33: c), a. raedera convergente, b. base de punta de proyectil triangular, c-e. especímenes bifaciales en distintas etapas de producción. 
(SU\#2). Creemos que la fecha de 7.440-7.620 años cal AP de la base de SU\#2 proporciona una edad mínima (i.e., terminus ante quem) para la superficie donde este artefacto se depositó. Esto también puede considerarse como una posible superficie para actividades al aire libre durante el Holoceno temprano (o anteriores) que dadas las condiciones de erosión local se encuentran actualmente expuestas.

Guiados por esta premisa, se condujeron nuevas exploraciones en Vega Coichel (campaña 2018), las que dieron como resultado el hallazgo de nuevas concentraciones superficiales de material lítico en el contorno de la cuenca. De éstas, destaca BN80 (45¹9'25'S; 71²1'14'O; 838 msnm). Se trata de una distribución cercana a los $1.400 \mathrm{~m}^{2}$ donde lascas de andesita con retoque marginal y otras sin modificaciones fueron registradas junto a una pequeña punta triangular de basalto fino. Una lasca retocada de andesita, de espesor medio y con remanentes de corteza, presenta evidencias de talla y retoque por ambos márgenes de una misma cara, y dos extracciones en el reverso para acondicionar la superficie de deslizamiento (Fig. 3a). Sus bordes activos rectos y abiertos y los ángulos producidos por la talla y el retoque permiten clasificarla como una raedera doble. La punta de proyectil, por su parte, se encuentra casi completa (Fig. 3b). Sus bordes (ligeramente convexos) son convergentes desde la base y ésta es de tipo convexa. Para su manufactura se alternó ambas caras en las fases de adelgazamiento, formatizado marginal y retoque.

La punta de proyectil registrada en BN80 se diferencia de otros hallazgos cercanos (e.g., BN44 y BN45) fechados en 1.370 a 1.510 años cal AP y de atributos típicamente tardíos, por la selección de las materias primas (no son calcedonias, ni sílices translúcidas a opacas) y la ausencia de atributos tecnomorfológicos que puedan asociarla a la manufactura de pequeños/medianos cabezales bifaciales triangulares pedunculados (Méndez et al. 2017). Respecto del artefacto de filo abierto, la materia prima seleccionada, su mayor tamaño y extensión del retoque, lo diferencian también.

\section{Comparaciones regionales y extra-regionales}

Los formatos triangulares bifaciales no son comunes en los conjuntos arqueológicos de PCO.
Un hallazgo superficial anterior ya había sido reportado en Appeleg 1 (Méndez et al. 2006) y una punta de proyectil intensamente reutilizada como raspador y muesca se registró asociada a una edad entre 5.520 y 5.400 años cal AP en El Chueco 1 (Méndez et al. 2011). Sin embargo, la pieza más similar a la descrita en BN80 por sus atributos (métricos y morfológicos) corresponde a una punta registrada en la cueva de Baño Nuevo 1 con una edad asociada entre 8.180 y 8.320 años cal AP (Mena et al. 2000). Piezas muy similares han sido registradas en Casa de Piedra de Roselló en el rango entre 8.580 y 8.400 años cal AP (Castro Esnal et al. 2017) y en Cerro Casa de Piedra 7 desde las primeras ocupaciones hacia $11.250 \mathrm{y}$ 10.730 años cal AP y hasta el Holoceno medio (Civalero, 2009). Similares morfologías habían sido descritas para el Holoceno temprano-medio del río Pinturas (Gradin et al. 1987).

Respecto a las piezas retocadas con filos largos en rocas de grano grueso como andesita y donde la formatización no altera mayormente la forma base (i.e., remanentes de corteza, retoque marginal a ultramarginal), éstas han sido registradas en la base de los sitios Cueva de la Vieja con una edad de 12.030 a 11.760 años cal AP y en El Chueco 1 con una edad de 11.650 a 11.240 años cal AP. En estos dos sitios, así como en Baño Nuevo 1, las evidencias de artefactos de filos largos más tardíos muestran mayor formatización y se encuentran manufacturados sobre rocas silíceas. Atributos similares son propios de las herramientas retocadas sobre lasca de módulos mediano-grande y grande registradas en los depósitos tempranos de lago Burmeister (Cerro Casa de Piedra 7) y río Pinturas (Cueva de las Manos 1) (Civalero, 2009).

\section{DISCUSIÓN Y COMENTARIOS}

El material lítico registrado recientemente en Appeleg 1 (CIS009; App 35-36) y en Vega Coichel (BN80) guarda similitudes tecno-estilísticas entre sî y se diferencia del material "típicamente" tardío registrado en las inmediaciones, tanto en la escala de sitio (CIS009), como de la localidad (BN44 y BN45). Las comparaciones regionales apuntan a que la combinación entre cabezales líticos de morfología triangular y lascas grandes con retoque marginal permitiría, al menos, excluir conjuntos 

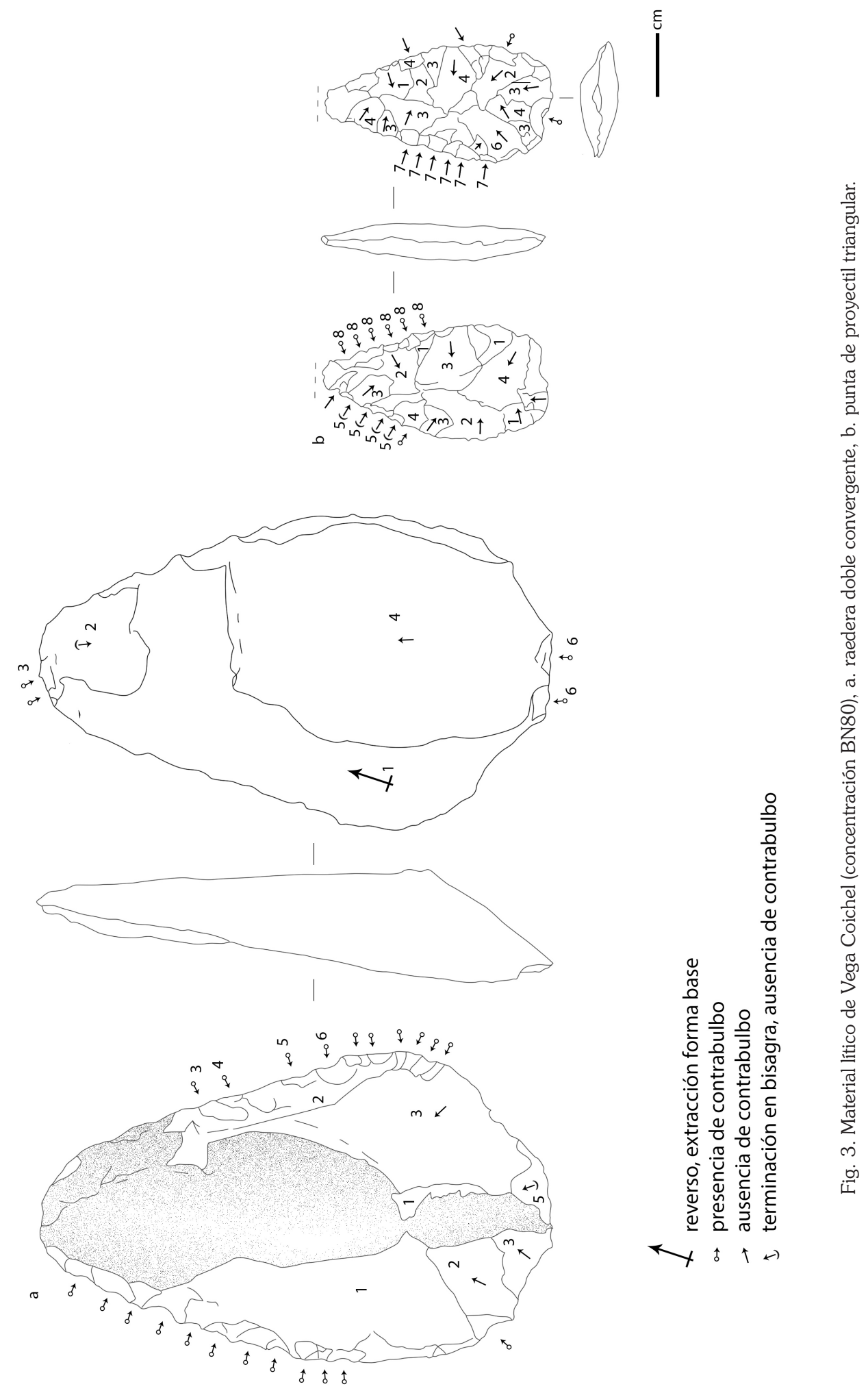
formados durante el Holoceno tardío, y como tales, pueden considerarse indicativos de dónde explorar superficies de ocupación más temprana (Nami \& Civalero, 2017). Tal asociación es muy poco frecuente en $\mathrm{PCO}$, lo que sería teóricamente consistente con una menor frecuencia de hallazgos de la primera mitad del Holoceno, ya sea por supervivencia diferencial, sedimentación o por una menor frecuencia de producción de material arqueológico en el espacio.

La evaluación de la evidencia humana temprana es un desafío complejo, ya que implica una mayor probabilidad de destrucción de ciertas unidades del paisaje y la pérdida tafonómica de líneas de evidencia arqueológica. En Patagonia, el reconocimiento de superficies potenciales para las ocupaciones del Holoceno temprano de Tierra del Fuego (McCulloch \& Morello, 2009; Morello et al. 2009) o la búsqueda de evidencia en localidades propensas a acumular restos paleontológicos y arqueológicos en las trampas volcánicas en Pali Aike (Martin \& San Román, 2010) son dos ejemplos de cómo distintos equipos de investigación han guiado búsquedas específicas. La implementación de dicha metodología ilustra que, al reconocer aspectos geográficos y ambientales y los procesos de formación que operan sobre el registro arqueológico, es factible apuntar hacia el descubrimiento de la evidencia humana en marcos cronológicos particulares. La alternativa por la que hemos optado en PCO es identificar geoformas de origen glaciar para excluir y dirigir zonas de búsqueda (Méndez et al. 2013). Esta aproximación ha sido combinada con análisis estratigráficos y la obtención de edades de unidades sedimentarias para asociar o descartar la ocurrencia de artefactos en bloques temporales específicos (Méndez et al. 2019).

Este estudio resume resultados preliminares obtenidos a través del programa diseñado para buscar vestigios arqueológicos en paisajes de la transición del Pleistoceno-Holoceno de PCO como una forma de distinguir las superficies que los primeros habitantes pudieron usar en sus actividades al aire libre. En este estudio, las consideraciones tecno-tipológicas y estilísticas se incluyeron como otro medio para orientar la búsqueda y luego como punto de comparación basada en el concepto de formas de hacer. Las asociaciones registradas deben considerarse como asociaciones espaciales y no necesariamente conductuales. La cercanía entre especímenes específicos o su disposición contigua sobre unidades estratigráficas expuestas en superficie sugiere potencial contemporaneidad. Sin embargo, esto debe ser tratado de manera hipotética en espera de líneas de evidencia alternativas que confirmen o refuten la asociación funcional.

Las edades radiocarbónicas en los dos sitios estudiados (Vega Coichel y Appeleg 1) son aún insuficientes y no se ha logrado establecer con claridad un marco temporal para los materiales de superficie, por lo que las observaciones deben considerarse preliminares. Potencialmente, sin embargo, éstas pueden indicar zonas donde los grupos humanos realizaron sus actividades $y$ cuáles fueron los entornos potenciales. Creemos que tal enfoque, aunque a veces sea lento $u$ ocasionalmente ingrato, puede proporcionar evidencias a largo plazo. El beneficio es que estos nuevos datos apuntan hacia incorporar mayor variabilidad al patrón dominante de registro arqueológico temprano bajo reparos rocosos, lo que sin duda ha proporcionado una visión aún incompleta respecto de los primeros habitantes de la Patagonia. Siguiendo a Borrero et al. (2007), así como a otros (e.g., Binford, 1978; Straus, 1990), la única manera de determinar la relevancia de las ocupaciones en cuevas es mediante la evaluación de su representatividad en los sistemas más amplios donde se originaron; $y$ eso es principalmente mediante la comprensión del uso de las localidades a cielo abierto.

\section{AGRADECIMIENTOS}

Financiado por el proyecto National Geographic HJ-150R-17, FONDECYT \#1180306 y CONICYT Programa Regional R17A10002. Las figuras 2 y 3 son producto de la colaboración entre los autores con Bárbara Thompson y Paulina Chávez. Stephanie Buckaert de Estancia Río Cisnes y Claudio Bariggi de Estancia Baño Nuevo otorgaron los permisos correspondientes para nuestras investigaciones. Agradecemos el apoyo en terreno de Bárbara Thompson, Manuela López, Pedro Fuentes, Joaquín Crisóstomo, Alejandra Jeldes y Gustavo Fredes y apoyo editorial de Javier 
Carranza. Agradecemos a Teresa Civalero, Darío Hermo, Enrique Terranova y a dos evaluadores anónimos por sus comentarios. Extendemos nuestro agradecimiento equipo editorial de Magallania.

\section{BIBLIOGRAFÍA}

Bate, L. F. (1974). Orígenes de la comunidad primitiva en Patagonia. D.F. México: Ediciones Cuicuilco, Escuela Nacional de Antropología e Historia.

Binford, L. R. (1978). Nunamiut Ethnoarchaeology. New York: Academic Press.

Bird, J. (1988). Travels and Archaeology in Southern Chile. Iowa City: University of lowa Press.

Borrero, L. A. (1989). Replanteo de la Arqueología Patagónica. Interciencia, 14(3), 127-135.

Borrero, L. A. (2001). Cambios, continuidades y discontinuidades: discusiones sobre arqueología FuegoPatagónica. En E. Berberián, \& A. Nielsen (Eds.), Historia argentina prehispánica (pp. 815-838). Córdoba: Editorial Brujas.

Borrero, L. A., \& Franco, N. V. (1997). Early Patagonian hunter-gatherers: subsistence and technology. Journal of Anthropological Research, 53, 219-239.

Borrero, L. A., Barberena, R., Martin, F. M., \& Borrazzo, K. (2007). Collapsed rockshelters in Patagonia. En M. Kornfeld (Eds.), Prés du bord d'un abri: Les histories, théories et méthodes de recherches sur les abris sous roche (pp. 135-139). BAR International Series 1655. Oxford: Archaeopress.

Borrero, L. A., Nuevo Delaunay, A., \& Méndez, C. (2019). Ethnographical and historical accounts for understanding the exploration of new lands: The case of Central Western Patagonia, Southernmost South America. Journal of Anthropological Archaeology, 54, 1-16.

Castro Esnal, A., Pérez de Micou, C. B., \& Casanueva, M. L. (2017). Early Holocene occupation of the forest-steppe ecotone of southern South America: evidence from Casa de Piedra de Roselló Cave (Chubut, Patagonia Argentina). PaleoAmerica, 3(3), 276-282.

Civalero, M. T. (2009). Tecnología lítica de cazadores tempranos en los contrafuertes de la Altiplanicie central santacruceña y el área lacustre cordillerana (lago Burmeister). En M. Salemme, F. Santiago, M. Álvarez, E. Piana, M. Vázquez \& M. E. Mansur (Eds.), Arqueología de Patagonia: Una mirada desde el último confín, tomo I (pp. 65-74). Ushuaia: Ediciones
Utopías.

Civalero, M. T., \& Franco, N. V. (2003). Early human occupations in western Santa Cruz province, southernmost South America. Quaternary International, 109-110, 77-86.

Collins, M. (1991). Rockshelters and the Early Archaeological Record in the Americas. En D. Meltzer \& T. Dillehay (Eds.), The First Americans. Search and Research (pp. 157-182). Boca Raton: CRC Press.

Contreras, C., Méndez, C., \& Reyes, O. (2016). Tecnología lítica de cazadores recolectores en la estepa aysenina. Gestión de recursos y organización espacial en los valles de los ríos Cisnes y Simpson. Magallania, 44(1), 167-185.

De Porras, M. E., Maldonado, A., Abarzúa, A. M., Cárdenas, M. L., Francois, J. P., Martel-Cea, A., Stern, C. R.,... \& Reyes, O. (2012). Postglacial vegetation, fire and climate dynamics at central Chilean Patagonia (Lake Shaman, $44^{\circ}$ S). Quaternary Science Reviews, 50, 7185.

De Porras, M. E., Maldonado, A., Quintana, F. A., Martel-Cea, A., Reyes, O., \& Méndez, C. (2014). Environmental and climatic changes in central Chilean Patagonia since the late Glacial (Mallin El Embudo, $44^{\circ} \mathrm{S}$ ). Climate of the Past, 10, 1063-1078.

Dillehay, T. D., Ramírez, C., Pino, M., Collins, M. B., Rossen, J., \& Pino-Navarro, J.D. (2008). Monte Verde: seaweed, food, medicine, and the peopling of South America. Science, 320, 784-786.

Frank, A. (2012). Los fogones en la meseta central de Santa Cruz durante el Pleistoceno final. Magallania, 40, 145162.

Gamble, C. (1999). The Palaeolithic societies of Europe. Cambridge: Cambridge University Press.

García, J.-L., Maldonado, A., de Porras, M. E., Nuevo Delaunay, A., Reyes, O., Ebensperger, C.A., Binnie, S.A.,... \& Méndez, C. (2019). Extensive early deglaciation and paleolake history of the Río Cisnes Ice Lobe, Patagonian Ice Sheet $44^{\circ}$ S. Quaternary Research, 91, 194-207.

Garreaud, R. D. (2009). The Andes climate and weather. Advances in Geosciences, 22, 3-11.

Goñi, R. (1995). Aleros: uso actual e implicancias arqueológicas. Cuadernos del Instituto Nacional de Antropología y Pensamiento Latinoamericano, 16, 329-341.

Gradin, C., Aschero, C., \& Aguerre, A. (1987). Primeros niveles culturales en el área río Pinturas. Estudios Atacameños, 8, 118-141.

Hermo, D., Terranova, E., Marchionni, L., Magnin, L., Mosquera, B., \& Miotti, L. (2013). Piedras o litos discoidales en Norpatagonia: evidencias en la meseta 
de Somuncurá (Río Negro, Argentina). Intersecciones en Antropología, 14, 507-513.

Hermo, D., Terranova, E., \& Miotti, L. (2015). Tecnología y uso de materias primas en puntas cola de pescado de la meseta de Somuncurá (Provincia de Río Negro, Argentina). Chungara, Revista de Antropología Chilena, 47(1), 101-115.

Inizan, M., Reduron-Ballinger, M., Roche, H., \& Tixier, J. (1999). Technologie de la pierre tailliée. Meudon Cedex: CREP.

Jackson, D. (2002). Los instrumentos líticos de los primeros cazadores de Tierra del Fuego. Santiago: DIBAM.

Jackson, D. (2007). Estructura, intensidad y reiteración en las ocupaciones paleoindias en cuevas y aleros de Patagonia meridional (Chile). Revista de Arqueología de Cazadores Recolectores del Cono Sur, 2, 67-87.

Jackson, D., \& Méndez, C. (2007). Litos discoidales tempranos en contextos de Patagonia. Magallania, 35(1), 75-84.

Jackson, D., Méndez, C., Seguel, R., Maldonado, A., \& Vargas, G. (2007). Initial occupation of the Pacific coast of Chile during Late Pleistocene times. Current Anthropology, 48(5), 725-731.

Luebert, F., \& Pliscoff, P. (2006). Sinopsis bioclimática y vegetacional de Chile. Santiago: Editorial Universitaria.

Martin, F. M., \& San Román, M. (2010). Explorando la variabilidad del registro arqueológico y tafonómico en Pali-Aike (Chile) a través de la búsqueda de registros Pleistocenos a cielo abierto. Magallania, 38(1), 199-215.

Martin, F. M., Todisco, D., Rodet, J., San Román, M., Morello, F., Prevosti, F., Stern, C. R., \& Borrero, L. A. (2015). Nuevas excavaciones en Cueva del Medio: procesos de formación de la cueva y avances en los estudios de interacción entre cazadores-recolectores y fauna extinta (Pleistoceno final, Patagonia Meridional). Magallania, 43(1), 165-189.

McCulloch, R. D., \& Morello, F. (2009). Evidencia glacial y paleoecológica de ambientes tardiglaciales y del Holoceno temprano. Implicaciones para el poblamiento temprano de Tierra del Fuego. En M. Salemme, F. Santiago, M. Álvarez, E. Piana, M. Vázquez \& M. E. Mansur (Eds.), Arqueología de Patagonia: una mirada desde el último confín, tomo I (pp.119-133). Ushuaia: Editorial Utopías.

Mena, F., \& Stafford, T. (2006). Contexto estratigráfico y fechación directa de esqueletos humanos del Holoceno temprano en cueva Baño Nuevo 1 (Patagonia Central, Chile). En J. Jiménez, S. González, J. Pompa \& F. Ortiz (Eds.), Segundo Simposio Internacional del Hombre Temprano en América (pp. 139-154). Ciudad de
México: INAH.

Mena, F., Lucero, V., Reyes, O., Trejo, V., \& Velásquez, H. (2000). Cazadores tempranos y tardíos en la cueva Baño Nuevo-1, margen occidental de la estepa centropatagónica. Anales del Instituto de la Patagonia, Serie Ciencias Humanas. 28, 173-195.

Méndez, C. (2013). Terminal Pleistocene/early Holocene 14C dates form archaeological sites in Chile: critical chronological issues for the initial peopling of the region. Quaternary International, 301, 60-73.

Méndez, C., Reyes, O., \& Velásquez, H. (2006). Tecnología lítica en el Alto Río Cisnes (estepa extra andina de la XI Región de Aisén): primeros resultados. Boletín de la Sociedad Chilena de Arqueología, 39, 87-101.

Méndez, C., Reyes, O., Nuevo Delaunay, A., Trejo, V., Barberena, R., \& Velásquez, H. (2011). Ocupaciones humanas en la margen occidental de Patagonia Central: eventos de poblamiento en Alto Río Cisnes. Magallania, 39(2), 223-242.

Méndez, C., Reyes, O., Nuevo Delaunay, A., \& González, P. (2013). Programa de búsqueda sistemática de evidencias tempranas y hallazgo de una punta de proyectil tipo cola de pescado en alto río Nirehuao. Magallania, 41(2), 187-196.

Méndez, C., de Porras, M.E., Maldonado, A., Reyes, O., Nuevo Delaunay, A., \& García, J.-L. (2016). Human effects in Holocene fire dynamics of central Western Patagonia ( $44^{\circ} \mathrm{S}$, Chile). Frontiers in Ecology and Evolution, 4, 100 .

Méndez, C., Reyes, O., Nuevo Delaunay, A., \& Latorre, E. (2017). Chenques en el centro oeste de Patagonia (Holoceno tardío final, valle de Nirehuao, $45^{\circ} \mathrm{S}$, Chile). Chungara, Revista de Antropología Chilena, 49(3), 379-395.

Méndez, C., Nuevo Delaunay, A., Reyes, O., Ozán, I. L., Belmar, C., \& López, P. (2018). The initial peopling of Central Western Patagonia (southernmost South America): late Pleistocene through Holocene site context and archaeological assemblages from Cueva de la Vieja site. Quaternary International, 473, 261-277.

Méndez, C., Nuevo Delaunay, A., Reyes, O., Maldonado, A., \& García, J.-L. (2019). A systematic strategy for assessing the early surface archaeological record of continental Aisén, Central Western Patagonia. En R. Suárez \& C. Ardelean (Eds.), People and Culture in Ice Age Americas, New Dimensions in Paleoamerican Archaeology (pp. 34-51). Salt Lake City: The University of Utah Press.

Miotti, L., \& Salemme, M.C. (2003). When Patagonia was 
colonized: people mobility at high latitudes during Pleistocene/Holocene transition. Quaternary International, 109-110, 95-111.

Morello, F., Borrero, L. A., Torres, J., Massone, M., Arroyo, M., McCulloch, R. D.,... \& Bahamonde, G. (2009). Evaluando el registro arqueológico de Tierra del Fuego durante el Holoceno temprano y medio. En M. Salemme, F. Santiago, M. Álvarez, E. Piana, M. Vázquez \& E. Mansur (Eds.), Arqueología de la Patagonia: Una mirada desde el último confín, tomo II (pp. 10311047). Ushuaia: Editorial Utopías.

Nami, H. G., \& Civalero, M.T. (2017). Distinctive unifacial technology during the Early Holocene in southern South America. Archaeological Discovery, 5, 101115.

Politis G., Gutiérrez, M. A., Rafuse, D. J., \& Blasi, A. (2016). The arrival of Homo sapiens into the Southern Cone at 14,000 years ago. PLoS ONE, 11(9), e0162870.

Prates, L., Politis, G., \& Steele, J. (2013). Radiocarbon chronology of the early human occupation of Argentina. Quaternary International, 301, 104-122.

Reyes, O., Méndez, C., Velásquez, H., \& Trejo, V. (2006). Distribuciones espaciales y contextos arqueológicos de cazadores recolectores esteparios en Alto río Cisnes (XI Región de Aisén). Magallania, 34(2), 75-90.

Sade, K. (2007). Cazadores extintos de Aysén continental: propuesta de poblamiento. Coyhaique: Ediciones Nire Negro.

Santoro, C., Ugalde, P., Latorre, C., Salas, C., Osorio, D., Jackson, D., \& Gayó, E. (2011). Ocupación humana pleistocénica en el Desierto de Atacama: primeros resultados de la aplicación de un modelo predictivo de investigación interdisciplinaria. Chungara, Revista de Antropología Chilena, 43, 353-366.

Straus, L. G. (1990). Underground archaeology: perspectives on caves and rockshelters. En M. B. Schiffer (Ed.), Archaeological Method and Theory 2 (pp. 255-304). Tucson: University of Arizona Press.

Suárez, M., De La Cruz, R., \& Bell, M. (2007). Geología del área Nireguao-Baño Nuevo. Región Aisén del General Carlos Ibáñez del Campo. Carta Geológica de Chile. Santiago: SERNAGEOMIN.

Suárez, R., Piñeiro, G., \& Barceló, F. (2018). Living on the river edge: The Tigre site (K-87) new data and implications for the initial colonization of the Uruguay River basin. Quaternary International, 473, 242-260.

Velásquez, H., Méndez, C., Reyes, O., Trejo, V., Sanhueza, L., Quiroz, D., \& Jackson, D. (2007). Campamentos residenciales tardíos a cielo abierto en el alto río Cisnes (XI Región de Aisén): Appeleg 1 (CIS 009). Magallania, 35(1), 85-98.

Waters, M., \& Stafford, T. (2013). The first Americans: a review of the evidence for the Late-Pleistocene peopling of the Americas. En K. Graf, C. Ketron \& M. Waters (Eds.), Paleoamerican Odyssey (pp. 543-562). College Station: Center for the Study of the First Americans.

Weitzel, C., Mazzia N., \& Flegenheimer, N. (2018). Assessing Fishtail points distribution in the southern Cone. Quaternary International, 473, 161-172. 